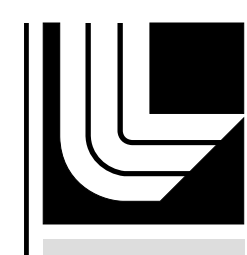

LAWRENCE LIVERMORE N A T IO N A L LABORATORY

\title{
Preparation of Single Cells for Imaging Mass Spectrometry
}

E. S. Berman, S. L. Fortson, K. S. Kulp, K. D. Checchi, L. Wu, J. S. Felton, K. J. Wu

October 29, 2007

Journal of the American Society for Mass Spectrometry 
This document was prepared as an account of work sponsored by an agency of the United States government. Neither the United States government nor Lawrence Livermore National Security, LLC, nor any of their employees makes any warranty, expressed or implied, or assumes any legal liability or responsibility for the accuracy, completeness, or usefulness of any information, apparatus, product, or process disclosed, or represents that its use would not infringe privately owned rights. Reference herein to any specific commercial product, process, or service by trade name, trademark, manufacturer, or otherwise does not necessarily constitute or imply its endorsement, recommendation, or favoring by the United States government or Lawrence Livermore National Security, LLC. The views and opinions of authors expressed herein do not necessarily state or reflect those of the United States government or Lawrence Livermore National Security, LLC, and shall not be used for advertising or product endorsement purposes. 


\title{
Preparation of Single Cells for Imaging Mass Spectrometry
}

\author{
Elena S.F. Berman, Susan L. Fortson, Kristen S. Kulp, Kyle D. Checchi, Ligang Wu, \\ James S. Felton, Kuang Jen J. Wu \\ Chemistry, Materials, and Life Sciences Directorate, Lawrence Livermore \\ National Laboratory, Livermore CA 94550
}

Running title: Preparation of Single Cells for Imaging MS

Address correspondence to E. Berman, Lawrence Livermore National Laboratory, 7000 East Ave, L-452, Livermore CA 94550. berman2@1lnl.gov 


\begin{abstract}
Characterizing chemical changes within single cells is important for determining fundamental mechanisms of biological processes that will lead to new biological insights and improved disease understanding. Imaging biological systems with mass spectrometry (MS) has gained popularity in recent years as a method for creating precise chemical maps of biological samples. In order to obtain high-quality mass spectral images that provide relevant molecular information about individual cells, samples must be prepared so that salts and other cell-culture components are removed from the cell surface and the cell contents are rendered accessible to the desorption beam. We have designed a cellular preparation protocol for imaging MS that preserves the cellular contents for investigation and removes the majority of the interfering species from the extracellular matrix. Using this method, we obtain excellent imaging results and reproducibility in three diverse cell types: MCF7 human breast cancer cells, Madin-Darby canine kidney (MDCK) cells, and NIH/3T3 mouse fibroblasts. This preparation technique allows routine imaging MS analysis of cultured cells, allowing for any number of experiments aimed at furthering scientific understanding of molecular processes within individual cells.
\end{abstract}

\title{
Introduction
}

In the past several years, there has been an explosion in the number of researchers utilizing imaging mass spectrometric techniques for biological applications [1-6]. While many of these have focused on the analysis of tissue samples, there is a clear need for analysis on a single-cell level as well. Individual cells are discrete packages of chemical information that can act as surrogates or sentinels for understanding responses of the whole organism. Currently, most biological analysis focuses on investigations of cellular populations. Results from these 
experiments necessarily average the response of all of the cells in the population, obscuring subtle phenotypic differences among individual cells that may provide mechanistic clues about biological processes [7]. By interrogating single cells, the analysis is freed from assumptions regarding cell population homogeneity and ensures that all potential cellular responses to environmental changes can be measured. Understanding the response of a single cell is necessary for identifying small cellular changes that may underlie many biological processes including disease development.

There are several different MS imaging systems currently being developed to analyze single cells. Although more commonly used for tissue analysis, recent advances in matrixassisted laser desorption/ionization (MALDI) MS imaging have greatly enhanced the imaging resolution, opening the possibilities for imaging of single cells $[8,9]$. To date, however, very few reports of single-cell MALDI imaging have been published [10]. Secondary ion mass spectrometry (SIMS), in both dynamic and static mode, has been widely applied to imaging analysis of single cells. Recent advances in dynamic SIMS imaging of cells have been reviewed elsewhere [4], with many examples showing excellent spatial resolution and localization of elemental species within cells. Very recently, the newest generation of dynamic SIMS instrumentation, NanoSIMS, has been used to obtain subcellular localization of a peptide vector [11], study diatom cell division [12], and perform nanoautography with stable isotope tracers [13]. Static SIMS has also seen wide application in cellular imaging. Interesting recent examples include three dimensional imaging of oocytes [14], relative quantification of cholesterol in cell membranes [15], and distinguishing cancerous cells of differing breast cancer phenotypes [16]. Cellular imaging has also been shown by a variety of other mass spectrometric 
techniques, including desorption/ionization on silicon [17] and laser post-ionization secondary neutral mass spectrometry [18].

We are utilizing time-of-flight secondary ion mass spectrometry (ToF- SIMS) to demonstrate the suitability and reproducibility of the reported cell preparation method for MS imaging. ToF-SIMS is a highly surface-sensitive, mass-spectral analysis technique used to detect and localize chemical and molecular information from sample surfaces. ToF-SIMS uses a finely focused ( $\sim 150 \mathrm{~nm})$, pulsed primary ion beam to desorb secondary ions into a time-offlight mass spectrometer. These secondary molecular and fragment ions are collected to create mass spectral images with excellent spatial resolution and good mass resolution. Each image consists of $256 \times 256$ pixels where each pixel is a complete mass spectrum. Mass spectral images can be analyzed as individual peaks or average mass spectra of a defined area.

As with any analytical technique, sample preparation is critical for meaningful and reproducible mass spectral imaging results. In order to obtain high-quality MS images of single cells, the cells must be attached on a suitable substrate, free of interfering components or contaminants, and accessible to the ionization source. Traditionally, for imaging mass spectral analysis, cells are grown on a conductive substrate and freeze fractured prior to analysis. The freeze-fracture technique was originally reported in 1957 and has been used extensively to prepare cells for membrane analysis by electron microscopy $[19,20]$. Working from this concept, Chandra and coworkers developed a modified freeze-fracture method that could be used to prepare cells for imaging MS analysis [21]. Currently, the vast majority of cellular MS imaging reports utilize some variation on this method. Improvements to Chandra's technique, most notably maintenance of the sample at cryogenic temperatures throughout the fracturing and analysis [22], have resulted in improvements to the cellular imaging analysis [15]. 
The freeze fracture method, however, has several disadvantages: it requires facilities for cryogenic preparation, generally produces a low yield of suitably fractured cells, and by design tends to fracture cells between the leaflets of the membrane bilayer. Because the fracture plane is most commonly within the membrane bilayer, the cytoplasm of the cell is still obscured by a layer of phospholipids. To circumvent these disadvantages and simplify cellular preparation, several groups have reported results with simpler wash and dry approaches. Liu and coworkers [17] chose to fix cells in $70 \%$ ethanol, which preserves the morphology of the cells but not the chemical contents of the cytosol. Parry and Winograd have reported embedding cells in a trehalose and glycerol matrix, followed by freeze-drying [23]. Altelaar and coworkers [24] used sucrose and water washes while Fletcher et al. [14] utilized only a water wash, both groups followed these washes with freeze-drying.

Here, we report development of a method that will both ensure that the full molecular information from the cytosol is retained and simplify previous procedures by removing the need for cryogenic facilities. We have found that the nature of both the wash and dry steps are critical to the quality of the mass spectral images produced. Components from cell-culture medium, most noticeably the salts, seriously interfere with the spectral quality making it nearly impossible to collect meaningful molecular information. Simply drying the cells onto a silicon substrate creates a coating of these interfering components, obscuring accessible mass spectral information. The simple "wash and dry" cellular preparation technique that we have developed not only successfully removes interferences from the substrate, but also allows delicate cells to remain intact until after all preparation steps are completed in order to obtain the greatest reproducible molecular information from each cell. 


\section{Experimental}

Cell Culture: MCF7 human breast adenocarcinoma cells, MDCK canine kidney cells, and NIH/3T3 mouse fibroblasts were obtained from American Type Culture Collection (ATCC, Manassas, VA). MCF7 cells were grown in DMEM (Dulbecco's Modified Eagle's Medium) with $5 \%$ fetal bovine serum (FBS), $1 \%$ nonessential amino acids, $10 \mu \mathrm{g} / \mathrm{mL}$ insulin, $2 \mathrm{mM} \mathrm{L-}$ glutamine, and 1\% penicillin/streptomycin. MDCK cells were grown in minimum essential medium (MEM, Eagle) with $2 \mathrm{mM}$ L-glutamine Earle's BSS, adjusted to contain $1.5 \mathrm{~g} / \mathrm{L}$ sodium bicarbonate, $0.1 \mathrm{mM}$ non-essential amino acids, $1.0 \mathrm{mM}$ sodium pyruvate, and 10\% FBS. $\mathrm{NIH} / 3 \mathrm{~T} 3$ cells were grown in DMEM with $4 \mathrm{mM}$ L-glutamine, adjusted to contain $1.5 \mathrm{~g} / \mathrm{L}$ sodium bicarbonate, $4.5 \mathrm{~g} / \mathrm{L}$ glucose and 10\% FBS. All culture reagents and media were obtained from Invitrogen Corporation (Carlsbad, CA). All cells were maintained at $37^{\circ} \mathrm{C}$ with $5 \% \mathrm{CO}_{2}$. Silicon substrates $\left(\sim 1.5 \mathrm{~cm}^{2}\right)$ were cleaned with $100 \%$ ethanol and sterilized by UV irradiation prior to experimentation. Approximately 15000 cells were plated in $60-\mathrm{mm}$ dishes containing 4 sterile silicon substrates using standard cell-culture techniques. The cells were allowed to attach overnight on the polished side of the silicon substrates. All these cells appear to grow equally well on the silicon substrates as on standard cell culture flasks showing no change in cellular morphology. In preparation for imaging mass spectrometry, each silicon wafer with attached cells was cleaned with one of a variety of methods as described in the results and discussion to remove cellular medium, particularly excess salts, and the associated mass spectral interferences. Samples were dried with argon as detailed below and stored for less than one week at room temperature and atmospheric pressure prior to ToF-SIMS analysis. 
Washing Solutions. Ammonium acetate, magnesium acetate, and Tris acid/Trizma base were purchased from Sigma (St. Louis, MO). Sucrose was purchased from J.T. Baker Inc. (Phillipsburg, NJ), sodium chloride was purchased from Mallinckrodt (Paris, KY), and HEPES (4-(2-hydroxyethyl)-1-piperazineethanesulfonic acid) was purchased from Invitrogen Corp. (Carlsbad, CA). The chemicals were individually dissolved to a concentration iso-osmotic to cellular cytosol (300-mM sucrose, 150-mM for all others) in Millipore Milli-Q water (18.2 $\mathrm{M} \Omega \mathrm{cm}$ ), the $\mathrm{pH}$ was adjusted to 7.5 using a 1:9 solution of phosphoric acid and $1 \mathrm{M}$ ammonium hydroxide, and each washing solution was sterile filtered using a Stericup® Vacuum Filter Cup from Millipore Corporation (Billerica, MA). Phosphate buffered saline (PBS) was made to be $0.137 \mathrm{M} \mathrm{NaCl}, 0.002 \mathrm{M} \mathrm{KCl}, 0.001 \mathrm{M} \mathrm{KH}_{2} \mathrm{PO}_{4}$, and $0.02 \mathrm{M} \mathrm{Na}_{2} \mathrm{HPO}_{4}$. All solutions were stored at room temperature under sterile conditions.

Cell Proliferation. MCF7 cells were plated at $2 \times 10^{4}$ cells/well in a 96-well plate and grown overnight at $37^{\circ} \mathrm{C}, 5 \% \mathrm{CO}_{2}$. Existing cell medium was removed from cells and $100 \mu 1$ of one washing solution was added and immediately removed. Fresh cell medium was then returned to the wells and the plate was placed back into the incubator for 48-hours to allow for cell proliferation. Cell growth was quantified using an Aqueous Non-Radioactive Cell Proliferation Assay (Promega, Madison WI) with absorbance of the wells measured in a standard multi-well plate reader (BIO-RAD Model 680 Microplate Reader) at 490nm. Briefly, this colormetric assay measures the bioreduction of a tetrazolium compound (3-(4,5-dimethylthiazol-2-yl)-5-(3carboxymethoxyphenyl)-2-(4-sulfophenyl)-2H-tetrazolium, inner salt; MTS) to a formazan product. The conversion of MTS is directly proportional to the number of living cells in culture. Each experiment was performed at least five individual times with 8 replicates per experiment. The effect of each washing buffer on cell growth was determined by comparing the washed wells 
to wells treated simply by removing medium and replacing with fresh medium. The data are presented as percent of control. Seventy percent ethanol is included as a negative control. Error is presented as the standard deviation of the mean proliferations from the five separate experiments. Statistical significance was tested using a one-sample Student's t-test (each condition compared to control) and a two-tailed t-test assuming unequal variances (ammonium acetate compared to water).

ToF-SIMS Analysis. ToF-SIMS measurements were conducted on a PHI TRIFT III instrument (Physical Electronics USA, Chanhassen, MN) equipped with a gallium $\left({ }^{69} \mathrm{Ga}^{+}\right)$liquid metal ion gun operated at $25 \mathrm{kV}$. Positive ion ToF-SIMS images were generally acquired over an area of $100 \times 100 \mu \mathrm{m}$ to $200 \times 200 \mu \mathrm{m}$ depending on the number and size of cells being imaged.

Samples were held at room temperature during the course of the ToF-SIMS measurements. All ToF-SIMS spectra were calibrated to the $\mathrm{CH}_{3}{ }^{+}, \mathrm{C}_{2} \mathrm{H}_{3}{ }^{+}$, and $\mathrm{C}_{3} \mathrm{H}_{5}{ }^{+}$peaks before further analysis.

\section{Results and Discussion}

The need for an efficient cell preparation method prior to imaging mass spectral analysis is illustrated in Figure 1 a. and b., which demonstrate the extent of the residue left on the substrates when cell medium is simply allowed to evaporate. Both an optical microscopic image of two MCF7 cells (Figure 1a.) and a ToF-SIMS total ion image of one MCF7 cell (Figure 1b.) show that the cells are completely surrounded and covered by cellular medium components, particularly salts. The optical image further shows that the cells are immediately surrounded by a ring-like halo of more concentrated medium components. Observation of cells as they dry has shown that during the drying process, liquid collects in the area immediately around cells, creating a droplet around each cell or group of cells after the surrounding substrate has dried. 
This droplet then evaporates, leaving dissolved components of the liquid solution concentrated in the area directly around the cells. This concentration of dissolved components, which interfere with direct imaging of the cells, compounds the challenge of sufficiently cleaning cellular samples.

In order to reduce the effect of this concentration of dissolved components, we have developed a process of blowing the substrates dry with a gentle and controlled argon stream (Figure 1c.). In this process, argon gas is directed at the sample substrate

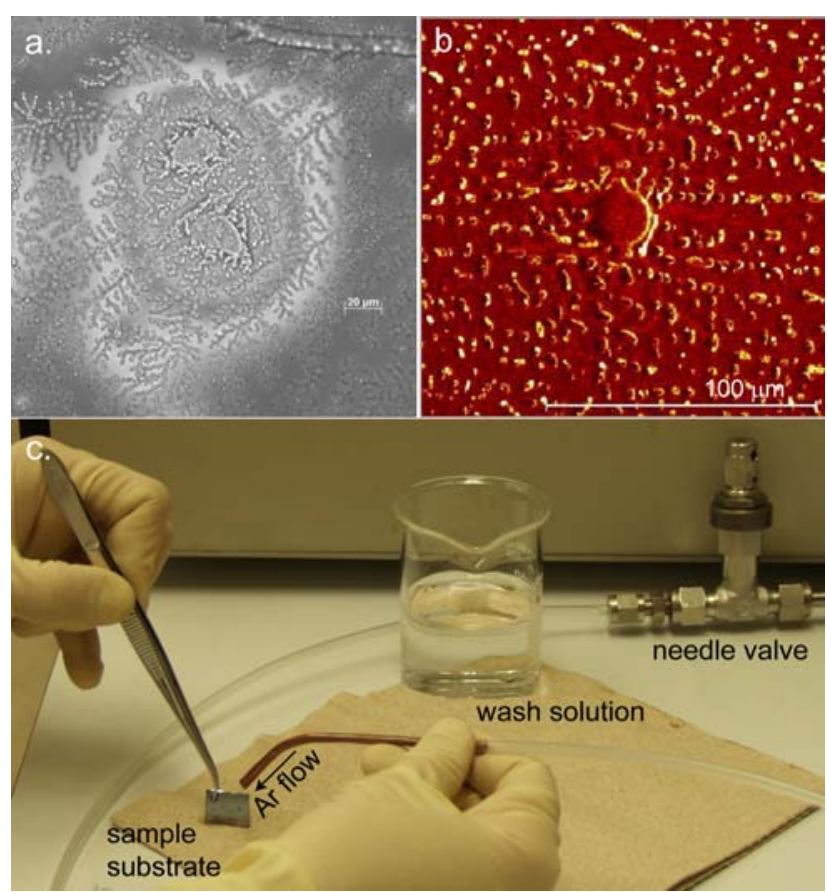

Figure 1. Optical 50x (a.) and ToF-SIMS total ion (b.) images of MCF7 human breast cancer cells grown on a silicon wafer and simply allowed to dry showing extreme contamination by salts and other medium components. Illustration (c.) of the experimental setup for quickly removing the solution from cells on a sample substrate using a gentle argon flow.

through a slit nozzle of approximately $1 \mathrm{~mm} \times 2 \mathrm{~mm}$ with an argon flow rate of $12-15 \mathrm{~cm}^{3} / \mathrm{s}$.

The nozzle is positioned so that the argon blows the liquid across the surface of the substrate and onto an absorbent towel that is perpendicular to one edge of the silicon substrate, thus significantly reducing the collection of liquid around the cells. We expect that any non-reactive gas which is clean and can be well controlled would be suitable for this drying step.

This drying process is designed to ensure that the liquid, along with any dissolved components, is pushed off of the substrate rather than evaporated, minimizing residue on the sample substrate. During the process, care must be exercised to avoid overly vigorous or prolonged treatment of the samples, which can cause loss of cellular integrity, specifically 
rupture of the cell membrane and loss of cytosolic contents. It should be noted that as with any process executed by hand, some day to day and operator-dependent variability is expected, but we have found it possible to obtain reproducibly good results with this technique.

While the aforementioned blowing technique reduces the amount of contamination observed in the area immediately around cells, it is not sufficient to render cells adequately clean for analysis. We have therefore undergone an extensive search for a washing solution which will both clean the cells sufficiently and preserve the maximum possible amount of cellular information for analysis. Recent investigations [14, 24] have reported using pure deionized water to wash cells prior to drying and imaging MS analysis. In our laboratory, however, we have found that washing with water, even for as few as 10 seconds, can cause a loss of cellular integrity during the washing step. Figure 2 shows the results from washing cells with water compared with those obtained using our optimized preparation technique described in detail below. Figure 2 a-c show total ion, sodium ion, and potassium ion images respectively of cells washed with pure water and blown dry. In ToF-SIMS images, the localization of sodium and potassium ions can be used as a general indicator of the location of the cytosol of a cell, as cytosol is known to contain a low concentration of sodium and a high concentration of potassium compared with the surrounding

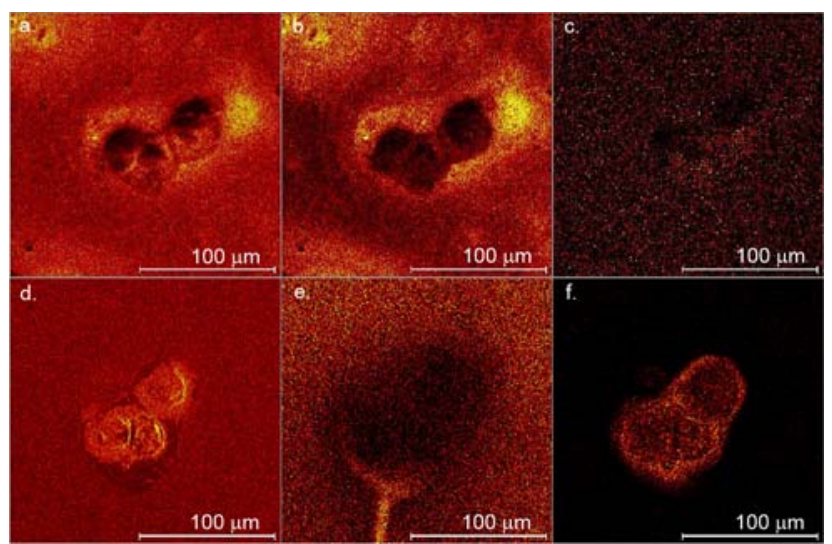

Figure 2. Row 1: ToF-SIMS images of MCF7 cells washed with deionized water: a. total ion image, b. sodium ion image showing exclusion from the cellular area, and c. potassium ion image showing a total lack of potassium localization in or near the cells. Row 2: ToF-SIMS images of similar MCF7 cells prepared with our reported optimized procedure: d. total ion image, e. sodium ion image showing exclusion from the cellular area and a small "tail" where the wash solution was blown away from the cells, and e. potassium ion image showing localization in the area just around the cells. 
medium [25]. In the water-washed cells, sodium is excluded from the cellular area as expected (Figure 2b), but there is a complete lack of potassium signal (Figure 2c), indicating that the soluble portion of the cytoplasm has likely been washed away from the cell body. As cells are known to rupture upon treatment with a hypo-osmotic solution [26], the most extreme of which is deionized water, it is reasonable to assume that the cells have exploded during the wash step, releasing the cellular contents to the wash solution, and allowing the cytosol to be washed away with the medium. These results are in contrast to those seen with our optimized preparation procedure, shown in Figure 2 d-f, where not only is sodium excluded from the cell region, but potassium is localized in the area immediately around the cells. These results suggest that, while water washing would be sufficient for analysis of the membrane and insoluble portions of a cell, an iso-osmotic wash solution is necessary to preserve the cellular cytosol and retain all the cytoplasmic information for MS imaging analysis.

A wide variety of iso-osmotic washing solutions were explored for their ability both to preserve cellular integrity through the washing process and to allow analysis by mass spectral imaging with the fewest imaging and spectral interferences. Possible candidates were chosen based on their use by others in the literature, (sucrose [24]), their widespread use in biological experiments, (PBS, Tris, HEPES and magnesium acetate), their simplicity (sodium chloride) or volatility (ammonium acetate). All solutions were created to be iso-osmotic with standard cellular medium. Various combinations of the above solutes were also evaluated for suitability although none of the combinations were found to provide better results than a single solute. The washing procedure involved quickly dipping the sample substrate into a small beaker of fresh solution, followed by swishing in one backward/forward motion. After washing, each sample 
substrate was dried with a gentle stream of argon as described above. The total time in contact with the wash solution was kept to less than 15 seconds.

The HEPES and magnesium acetate solutions were found to be unsuitable because the ToF-SIMS spectra of cells prepared with these solutions contained large mass spectral peaks associated with the washing solution.

Figure 3 shows representative imaging results from testing the washing solutions. As can be seen in panels a and b, PBS improved the cleanliness of the cells over medium alone, but still left a large number of salt crystals on the sample substrate.

Washing with sodium chloride produced similar results. The sucrose wash (Figure 3, c and d) eliminated the problem of salts interfering with MS imaging, but unfortunately left a sticky residue on the sample substrate which is clearly visible in the ToF-SIMS images. By far the best washing solution was found to be $150-\mathrm{mM}$ ammonium acetate, as can be seen from the optical and ToF-SIMS images in Figure 3, e and $\mathrm{f}$. Ammonium acetate washing followed by gentle blowing produces large,

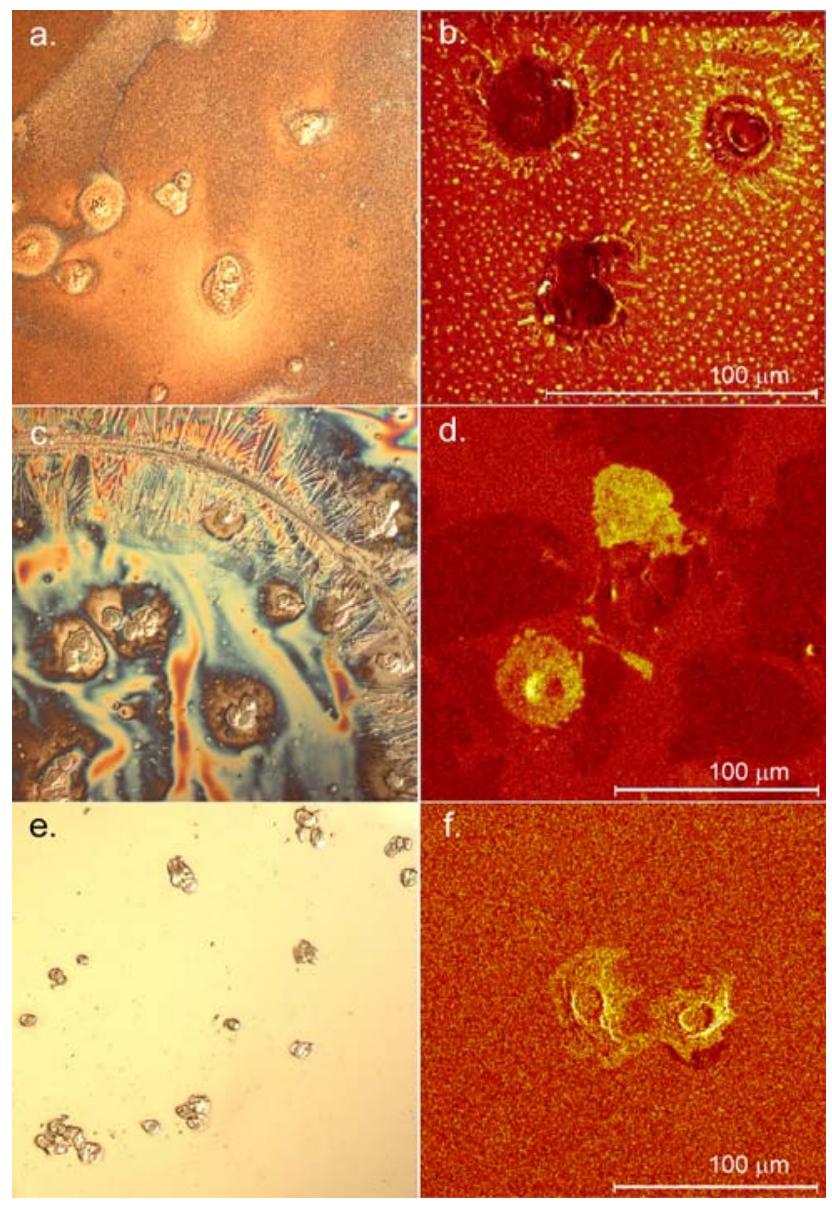

Figure 3. MCF7 cells washed with various isoosmotic solutions and blown dry for imaging mass spectral analysis. Optical 20x (a) and ToF-SIMS total ion (b) images of cells washed with PBS showing significant contamination by salts. Optical 20x (c) and ToF-SIMS total ion (d) images of cells washed with sucrose showing interference from remaining sugar. Optical 20x (e) image of cells washed with ammonium acetate showing clean silicon between cells and ToF-SIMS total ion (f) image of cells showing no interference from salts or other medium or wash components. 
bright areas of clean silicon surrounding the cells in the optical image and the complete absence of salt crystals and other interference in the ToF-SIMS total ion image. The superior results obtained with the ammonium acetate wash likely are due to the relative volatility of ammonium acetate, which allows any residue remaining after the drying procedure to evaporate rather than interfere with mass spectral analysis.

However, as was made evident by the problems created by washing with pure water, a washing procedure must maintain cellular integrity in addition to removing spectral and imaging interferences. We have used a standard cellular proliferation assay to measure the effects of the various washing solutions on cell viability. Figure 4 shows the effects of three of the washing solutions and the negative control $(70 \%$ ethanol) on MCF7 cell growth. As expected with any disruption of the cells, all the treatments show some decrease in the number of cells relative to the control. Treatment with water shows a statistically significant $(\mathrm{p}<0.0001)$ decrease in viable cells, as expected based on cellular physiology and previous ToF-SIMS

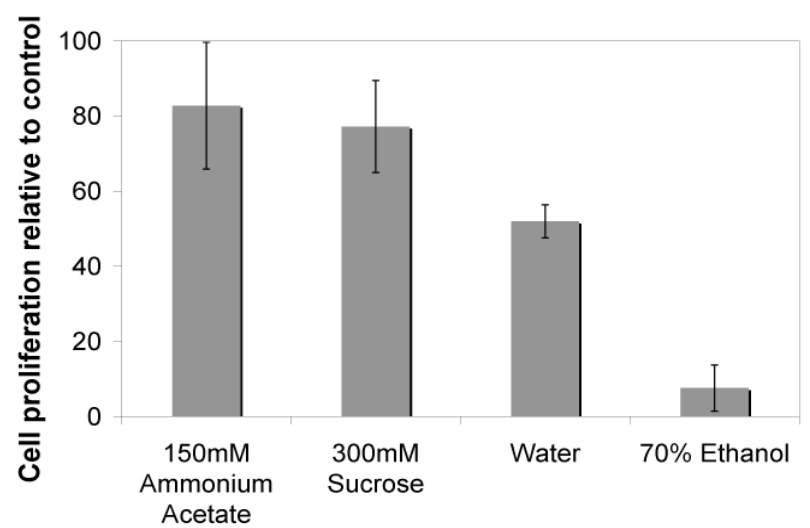

Figure 4. Average effect of washing solutions on MCF7 cell proliferation. Cells were washed with various solutions and allowed to grow for 48 hours. Data are normalized to the growth of cells that were not washed (cellular medium). Error bars represent the standard deviation of the mean proliferations from the five separate experiments.

experiments. Treatment with ammonium acetate causes a minimal decline in the number of viable cells, indicating that washing with ammonium acetate is indeed the best washing procedure both from the standpoint of keeping the cells intact during washing and for producing clean cells for imaging analysis. It is interesting to note that washing with water does not cause a total reduction in cell growth when compared to the negative control ( $70 \%$ ethanol treatment). 
These results may indicate why this technique has been reported as successful by others [14, 24]. However, the statistically significantly $(\mathrm{p}=0.013)$ better viability obtained with the ammonium acetate, in addition to the excellent imaging characteristics, indicate that washing with ammonium acetate is preferable to washing with water for imaging MS analysis of cells.

It is important to note that while it is critical to maintain cellular integrity during any washing procedure in order to preserve the cytosolic contents of the cell, a dried cell will, by definition, not be entirely comparable to a living cell. Our washing and drying procedure has been designed to maintain an intact cell throughout the washing and blowing steps. However, after drying, as the surrounding solution has been blown away, a high pressure differential will be created between the still-hydrated cell and the surrounding air. This pressure differential will cause the membrane to lose structure and collapse on the cell body. During this process, the cytosol leaks into the immediate vicinity of the cell. As long as this leakage occurs after all washing and drying procedures are completed, the entire cellular contents are retained in the area immediately surrounding the cell and available for mass spectral analysis. This technique has the advantage of preserving all the chemical information, albeit without specific information on fine localization within the cytoplasm.

A critical component of any biological sample preparation is reproducible results. To address this issue, we repeatedly prepared and analyzed MCF7 cells over a one-month period. Figure 5 shows ToF-SIMS images and mass spectra from $m / z=100$ to 220 for four MCF7 cells prepared on four different silicon substrates. From these results it is evident that the optimized cellular preparation procedure produces highly reproducible results. 

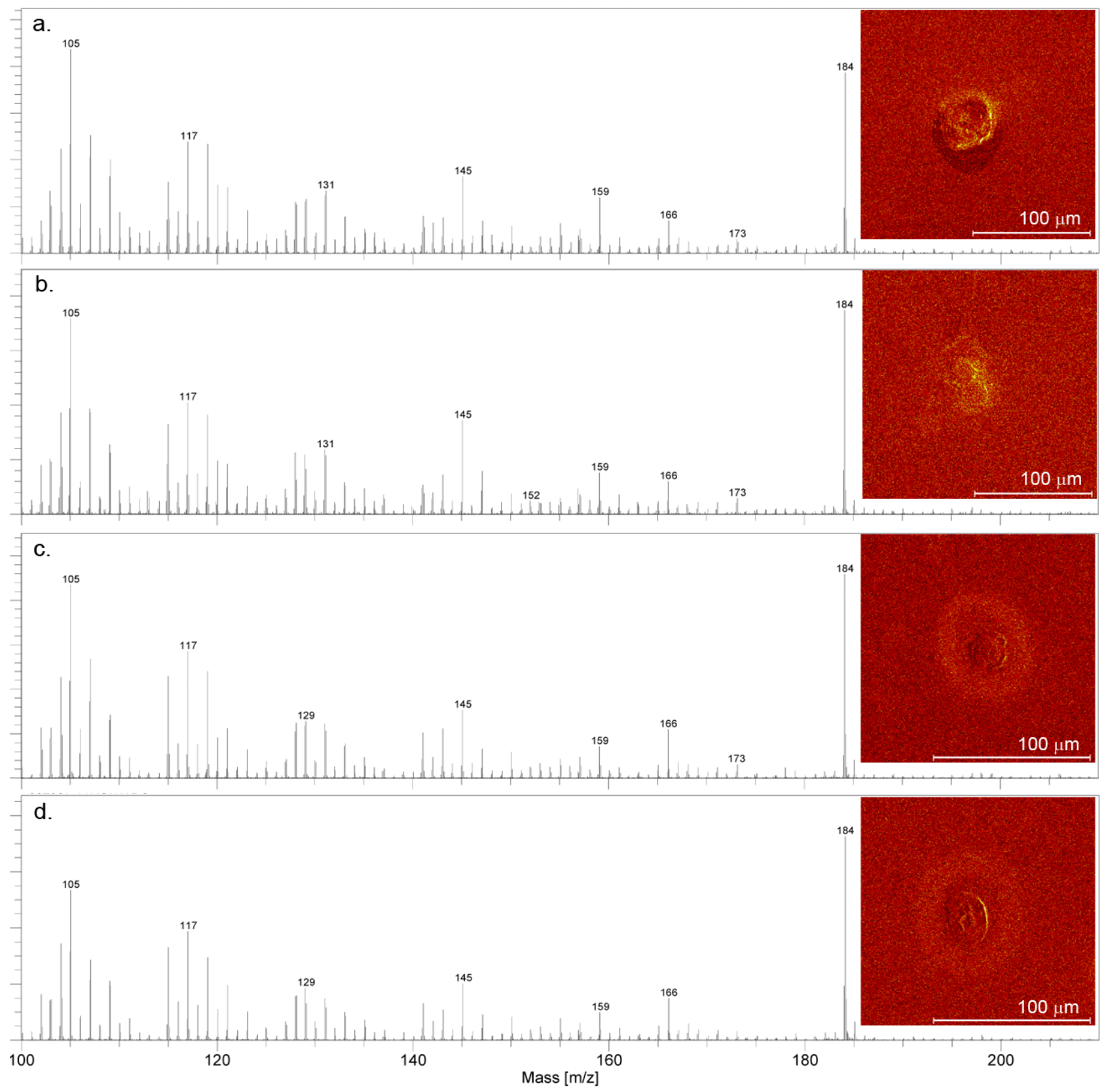

Figure 5. ToF-SIMS spectra and total ion images of four MCF7 human breast cancer cells prepared on four different substrates over a period of one month showing the reproducibility attained with the reported preparation method. 
In addition, this preparation

procedure is applicable to a wide variety of cell types. Figure 6 shows ToF-SIMS images taken from seven MCF7 human breast cancer cells (a and b), two whole MDCK cells (c and d), and one NIH/3T3 mouse fibroblast (e and f). These cells, which represent different species, different organs, and different states of differentiation, are widely used models for studying various biological processes.

These include the role of estrogen in breast cancer (MCF7), the function of polarized epithelial cells (MDCK), the processing of beta-amyloid precursor protein (MDCK), intercellular communication (3T3) and oncogenic transformation (3T3). The images in Figure 6 demonstrate that, while the cells

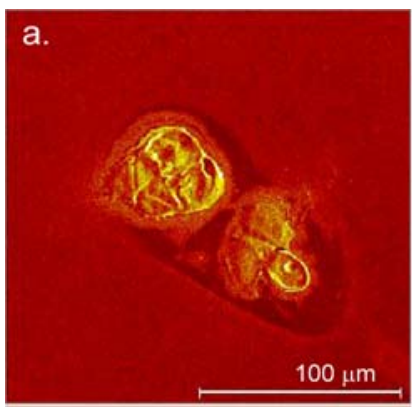

\section{b.}

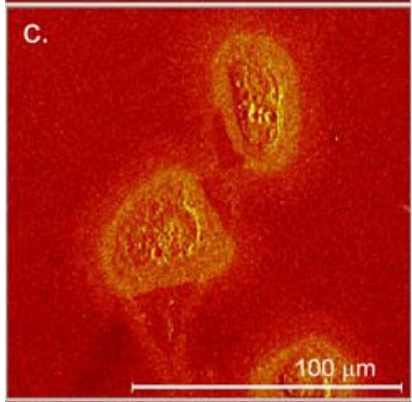

d.

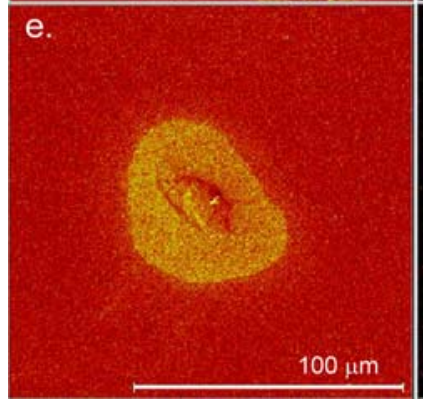

f.

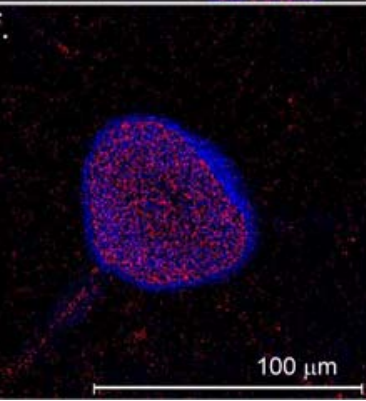

Figure 6. ToF-SIMS images of three different cell types prepared with the reported optimized cellular preparation procedure. In the left column are total ion images, in the right column composite images of potassium ion (blue) and $m / z=184$, a fragment of the phosphocholine head group (red). $a$. and b.: MCF7 human breast cancer cells, c. and d.: MDCK canine kidney cells, and e. and f.: NIH/3T3 mouse fibroblast. Note the excellent localization of potassium in the area immediately surrounding the cells and the localization of phophocholine on the cellular region.

clearly differ in morphology, the preparation procedure produces cells suitable for detailed imaging analysis for each cell type, making this a broadly applicable procedure for a variety of biological investigations.

In Figure 6, the left column shows total-ion images of the cells, while the right column shows overlay single-ion images of the potassium ion (blue) and $m / z=184$, a fragment of the 
phosphocholine head group. The overlay images clearly illustrate the localization of phosphocholine on the membrane areas and potassium from the cytosol in the area immediately surrounding the cell bodies. This figure illustrates the excellent images achievable with ToFSIMS, with both membrane and cytosolic regions clearly visible.

Figure 6 highlights an additional benefit of the cellular preparation procedure described herein. The localization shown in the overlay images suggests that after the quick washing and drying procedure, the cytosol of the cell is contained within the immediate area around the cell. Based on the excellent localization of potassium, we believe that the membrane collapse and release of cytosol is occurring after all washing and drying procedures, thus retaining the cytosol in the area around the cell and making it available for single cell imaging MS analysis.

The potassium localization can also be used as an indicator for correct washing and drying procedures. If the washing step is too vigorous or too long, the potassium signal will be lost, while if the drying step is too harsh the potassium signal from the cytosol will be seen as a tail leaving the cell where it has been blown away by the argon stream. Most importantly, the potassium localization indicates that the cytosol of the cells has been rendered available for MS analysis, while the phosphocholine signal indicates that the membrane portions are also being analyzed.

\section{Conclusions}

These experiments have shown that the reported cellular preparation method both preserves the molecular information from single cells and produces sufficiently clean surfaces for unobscured mass spectral imaging analysis. Furthermore, this preparation technique yields reproducible results over separate preparations, is applicable to a wide variety of cell types, removes the need 
for cryogenic facilities and produces a high yield of cells suitable for imaging. Importantly, the quick drying step also allows for the interrogation of both membrane and cytosolic molecular contents, as evidenced by excellent localization of both phosphocholine and potassium in the mass spectral images. The reported preparation technique allows routine imaging MS analysis of individual cells, opening the possibilities for a large variety of experiments aimed at furthering scientific understanding of chemical processes within cells.

\section{Acknowledgements}

The authors gratefully acknowledge the assistance of Cynthia Thomas in culturing cells and Donald J. Sirbuly for microscopy. This work was performed under the auspices of the U.S. Department of Energy by Lawrence Livermore National Laboratory in part under Contract No. W-7405-Eng-48 and in part under Contract DE-AC52-07NA27344 and supported in part by BCRP 10IB-0077, 11NB-0178 and LDRD 04-ERD-104 (LLNL internal funding). 


\section{References}

1. Belu, A. M.; Graham, D. J.; Castner, D. G., Time-of-Flight Secondary Ion Mass Spectrometry: Techniques and Applications for the Characterization of Biomaterial Surfaces. Biomaterials 2003, 24, 3635-3653.

2. Lockyer, N. P.; Vickerman, J. C., Progress in Cellular Analysis Using Tof-Sims. Appl. Surf. Sci. 2004, 231-232, 377-384.

3. Rubakhin, S. S.; Jurchen, J. C.; Monroe, E. B.; Sweedler, J. V., Imaging Mass Spectrometry: Fundamentals and Applications to Drug Discovery. Drug Discovery Today 2005, 10, 823-837.

4. Guerquin-Kern, J.-L.; Wu, T.-D.; Quintana, C.; Croisy, A., Progress in Analytical Imaging of the Cell by Dynamic Secondary Ion Mass Spectrometry (Sims Microscopy). Biochim. Biophys. Acta. 2005, 1724, 228-238.

5. Chaurand, P.; Cornett, D. S.; Caprioli, R. M., Molecular Imaging of Thin Mammalian Tissue Sections by Mass Spectrometry. Curr. Opin. Biotechnol. 2006, 17, 431-436.

6. $\quad$ Reyzer, M. L.; Caprioli, R. M., Maldi-Ms-Based Imaging of Small Molecules and Proteins in Tissues. Curr. Opin. Chem. Biol. 2007, 11, 29-35.

7. Elowitz, M. B.; Levine, A. J.; Siggia, E. D.; Swain, P. S., Stochastic Gene Expression in a Single Cell. Science 2002, 297, 1183-1186.

8. Luxembourg, S. L.; Mize, T. H.; McDonnell, L. A.; Heeren, R. M. A., High-Spatial Resolution Mass Spectrometric Imaging of Peptide and Protein Distributions on a Surface. Anal. Chem. 2004, 76, 5339-5344.

9. Jurchen, J. C.; Rubakhin, S. S.; Sweedler, J. V., Maldi-Ms Imaging of Features Smaller Than the Size of the Laser Beam. J. Am. Soc. Mass Spectrom. 2005, 16, 1654-1659.

10. Rubakhin, S. S.; Greenough, W. T.; Sweedler, J. V., Spatial Profiling with Maldi Ms: Distribution of Neuropeptides within Single Neurons. Anal. Chem. 2003, 75, 5374-5380.

11. Romer, W.; Wu, T.-D.; Duchambon, P.; Amessou, M.; Carrez, D.; Johannes, L.; Guerquin-Kern, J.-L., Sub-Cellular Localisation of a ${ }^{15}$ n-Labelled Peptide Vector Using Nanosims Imaging. Appl. Surf. Sci. 2006, 252, 6925-6930.

12. Audinot, J.-N.; Guignard, C.; Migeon, H.-N.; Hoffmann, L., Study of the Mechanism of Diatom Cell Division by Means of ${ }^{29}$ si Isotope Tracing. Appl. Surf. Sci. 2006, 252, 68136815.

13. McMahon, G.; Glassner, B. J.; Lechene, C. P., Quantitative Imaging of Cells with MultiIsotope Imaging Mass Spectrometry (Mims) - Nanoautography with Stable Isotope Tracers. Appl. Surf. Sci. 2006, 252, 6895-6906.

14. Fletcher, J. S.; Lockyer, N. P.; Vaidyanathan, S.; Vickerman, J. C., Tof-Sims 3d Biomolecular Imgaging of Xenopus Laevis Oocytes Using Buckminsterfullerene $\left(\mathrm{C}_{60}\right)$ Primary Ions. Anal. Chem. 2007, 79, 2199-2206.

15. Ostrowski, S. G.; Kurczy, M. E.; Roddy, T. P.; Winograd, N.; Ewing, A. G., Secondary Ion Ms Imaging to Relatively Quatify Cholesterol in the Membranes of Individual Cells from Differentially Treated Populations. Anal. Chem. 2007, 79, 3554-3560.

16. Kulp, K. S.; Berman, E. S. F.; Knize, M. G.; Shattuck, D. L.; Nelson, E. J.; Wu, L.; Montgomery, J. L.; Felton, J. S.; Wu, K. J., Chemical and Biological Differentiation of Three Human Breast Cancer Cell Types Using Time-of-Flight Secondary Ion Mass Spectrometry (Tof-Sims). Anal. Chem. 2006, 78, 3651-3658. 
17. Liu, Q.; Guo, Z.; He, L., Mass Spectrometry Imaging of Small Molecules Using Desorption/Ionization on Silicon. Anal. Chem. 2007, 79, 3535-3541.

18. Arlinghaus, H. F.; Kriegeskotte, C.; Fartmann, M.; Wittig, A.; Sauerwein, W.; Lipinsky, D., Mass Spectrometric Characterization of Elements and Molecules in Cell Cultures and Tissues. Appl. Surf. Sci. 2006, 252, 6941-6948.

19. Wells, W. A., The Invention of Freeze Fracture Em and the Determination of Membrane Structure. The Journal of Cell Biology 2005, 168, 174-175.

20. Steere, R. L., Electron Microscopy of Structural Detail in Frozen Biological Specimens. Journal of Biophysical and Biochemical Cytology 1957, 3, 45-60.

21. Chandra, S.; Morrison, G. H., Sample Preparation of Animal Tissues and Cell Cultures for Secondary Ion Mass Spectrometry (Sims) Microscopy. Biology of the Cell 1992, 74, 31-42.

22. Cannon, D. M. J.; Pacholski, M. L.; Winograd, N.; Ewing, A. G., Molecule Specific Imaging of Freeze-Fractured, Frozen-Hydrated Model Membrane Systems Using Mass Spectrometry. J. Am. Chem. Soc. 2000, 122, 603-610.

23. Parry, S.; Winograd, N., High-Resolution Tof-Sims Imaging of Eukaryotic Cells Preserved in a Trehalose Matrix. Anal. Chem. 2005, 77, 7950-7957.

24. Altelaar, A. F. M.; Klinkert, I.; Jalink, K.; de Lange, R. P. J.; Adan, R. A. H.; Heeren, R. M. A.; Piersma, S. R., Gold-Enhanced Biomolecular Surface Imaging of Cells and Tissue by Sims and Maldi Mass Spectrometry. Anal. Chem. 2006, 78, 734-742.

25. Stryer, L., Biochemistry. 2nd ed.; W.H. Freeman and Company: San Francisco, 1981.

26. Cambell, N. A., Biology. 3rd ed.; The Benjamin/Cummings Publishing Company, Inc.: Redwood City, California, 1993. 